Supporting Information

\title{
Plasmonic Switching: Hole Transfer Opens an Electron- Transfer Channel in Plasmon-Driven Reactions
}

\author{
Dokyung Lee and Sangwoon Yoon* \\ Department of Chemistry, Chung-Ang University, 84 Heukseok-ro, Dongjak-gu, Seoul 06974, \\ Korea. \\ *E-mail: sangwoon@cau.ac.kr
}




\section{Materials}

The following materials were used as received. From Sigma Aldrich: 4-mercaptobenzoic acid (MBA, 99\%), benzenethiol (BT, 99.0\%), 4-aminobenzenethiol ( $\mathrm{ABT}, 97 \%)$, 1-pentanethiol ( $\left.\mathrm{C}_{5} \mathrm{SH}, 98 \%\right)$, 6mercaptohexanoic acid (MHA, 90\%), 4-mercaptophenol (MP, $\geq 97 \%)$, sodium borohydride $\left(\mathrm{NaBH}_{4}, \geq 98 \%\right)$, dimethylformamide (DMF, 99.8\%) and sodium hydroxide ( $\mathrm{NaOH}, \geq 98 \%)$. From other suppliers: 4mercaptobenzyl alcohol ( $\mathrm{MBnOH}, \geq 90 \%$, Santa Cruz Biotechnology), 4-nitrobenzenethiol (NBT, $\geq 98 \%$, Santa Cruz Biotechnology), hydrochloric acid $\left(\mathrm{HCl}, 35.0-37.0 \%\right.$, Duksan Chemical), sulfuric acid $\left(\mathrm{H}_{2} \mathrm{SO}_{4}\right.$, $>95 \%$, Duksan Chemical), hydrogen peroxide $\left(\mathrm{H}_{2} \mathrm{O}_{2},>34.5 \%\right.$, Daejung Chemical), bare AuNPs (51 nm (nominally $45 \mathrm{~nm}$ ), Cytodiagnostics), ethanol (anhydrous, 99.9\%, Samchun) and water (HPLC grade, J. T. Baker).

\section{Size of AuNPs}

(a)

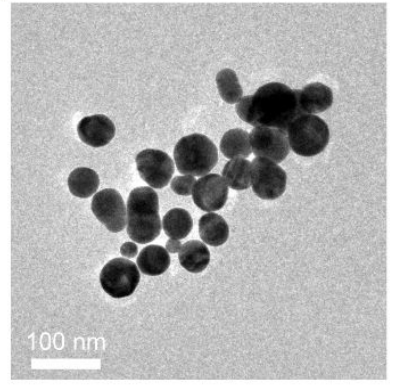

(b)

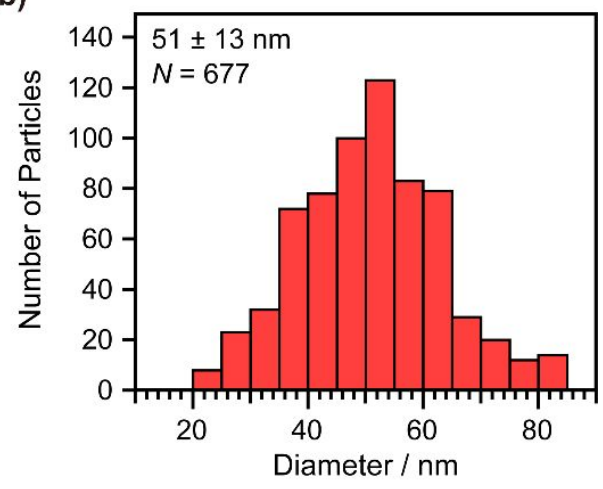

Figure S1. (a) Representative transmission electron microscopy (TEM, JEM-F200, JEOL) image of the AuNPs used in our experiments. (b) Size distribution of the measured AuNPs.

\section{Density of AuNPs on SAMs}
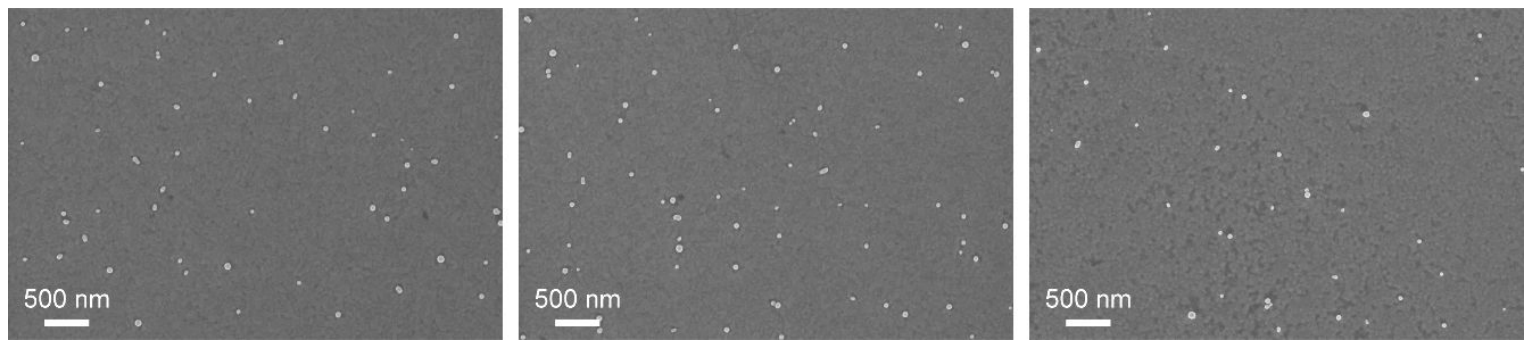
Figure S2. Examples of scanning electron microscopy (SEM, Sigma, Carl Zeiss) images acquired from the NPoM samples in our experiments. The average number of AuNPs within the focal area (diameter $=10 \mu \mathrm{m}$ ) corresponds to $253 \pm 32$.

\section{Reactivity of NBT in Nanogaps between AuNPs and Au Films under Acidic, Neutral, and Basic} Conditions upon Plasmon Excitation

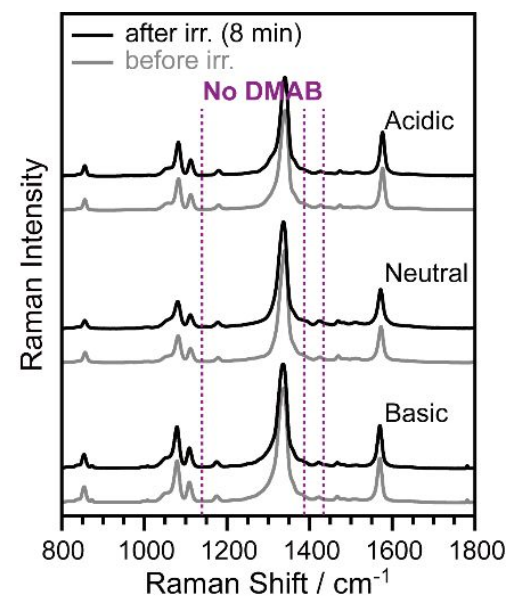

Figure S3. SERS spectra of NBT SAMs between AuNPs and Au films before (gray lines) and after (black lines) irradiation at $785 \mathrm{~nm}$ for $8 \mathrm{~min}$. No peaks attributable to DMAB (purple dotted lines) are observed after irradiation, indicating that the plasmon-driven reduction of NBT to DMAB does not occur for NBT SAMs in Au nanogaps, regardless of the $\mathrm{pH}$ conditions. 


\section{Reduction of NBT to DMAB upon Addition of $\mathrm{NaBH}_{4}$ in NPoM}

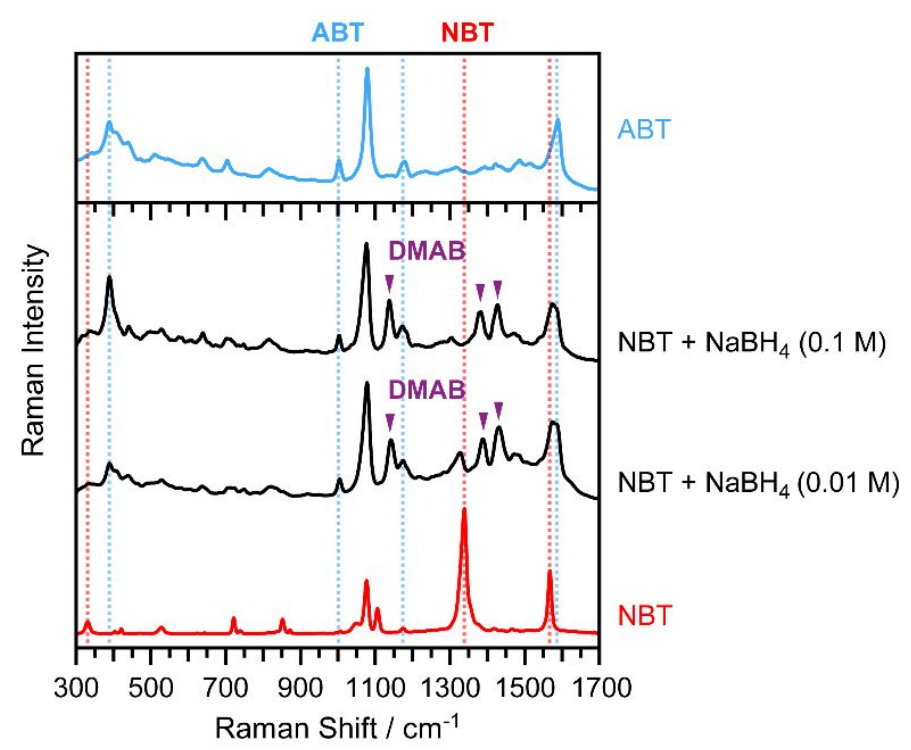

Figure S4. Plasmon-driven reduction of NBT to DMAB (and further to $A B T$ ) requires a reducing agent $\left(\mathrm{NaBH}_{4}\right)$. The SERS spectrum of NBT from the SAMs in NPoM evolves as $\mathrm{NaBH}_{4}$ is added. The SERS peaks attributed to DMAB (purple triangles) and even ABT (cyan dotted lines) appear. The ABT peaks are more pronounced (see peak near $\left.400 \mathrm{~cm}^{-1}\right)$ as more concentrated $\mathrm{NaBH}_{4}(0.1$ $M)$ is added. The SERS spectrum of ABT from NPoM is included for comparison (cyan solid line).

\section{Comparison of Raman Scattering Cross-Sections between NBT and MBA}

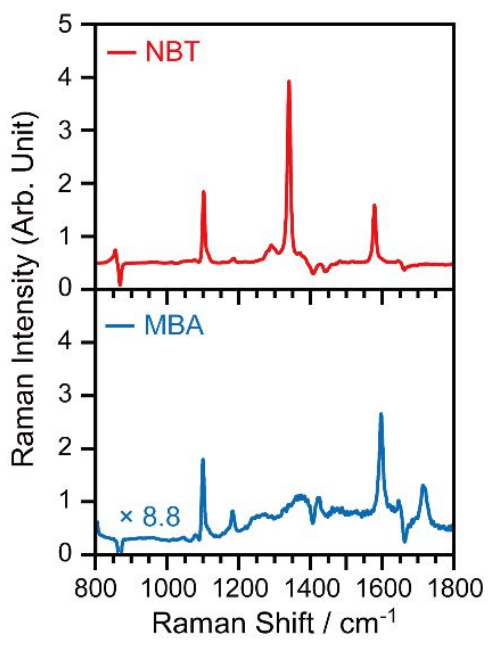

Figure S5. Normal Raman spectra of NBT and MBA. NBT (0.465 M) and MBA (0.246 M) were dissolved in DMF. Raman spectra were acquired from a capillary containing each solution $\left(\lambda_{\text {exc }}\right.$ 
$=785 \mathrm{~nm}$, power at sample $=50 \mathrm{~mW}$, total exposure time $=20 \mathrm{~s})$, and then the spectrum of the pure solvent was subtracted. The Raman scattering cross-section of NBT is $\sim 9$ times larger than that of MBA with respect to the $\delta_{\mathrm{ccc}}$ mode at $1079 \mathrm{~cm}^{-1}$.

\section{NBT-to-DMAB Reaction in Mixed SAMs with Different Mixing Ratios}

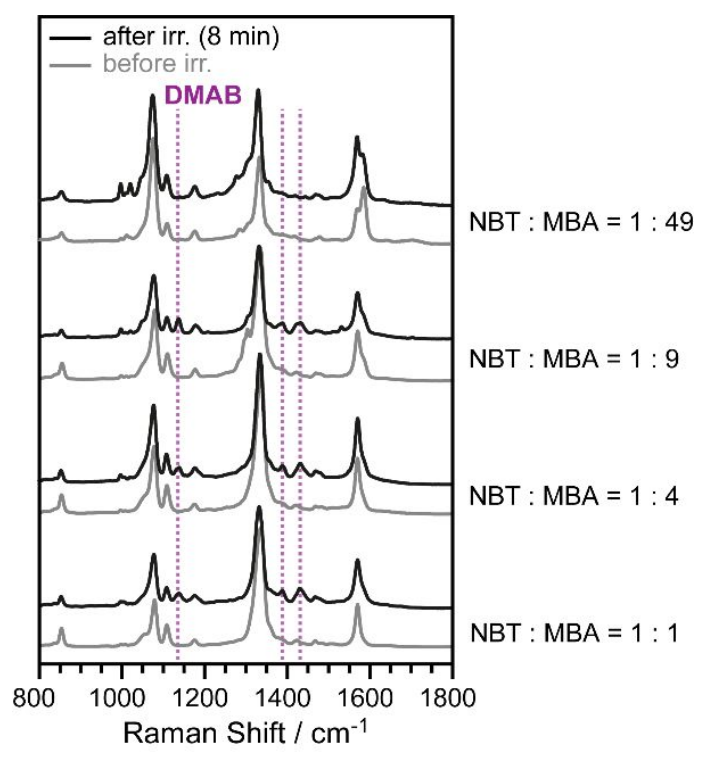

Figure S6. Representative SERS spectra of NBT + MBA mixed SAMs in NPOM in different mixing ratios, before (gray lines) and after (black lines) irradiation at $785 \mathrm{~nm}$. The DMAB products (purple dashed lines) from the reduction of NBT are observed in the presence of MBA in the SAMs in mole ratios of 1:1, 1:4, and 1:9. When NBT is too diluted (NBT:MBA = 1:49), the dimerization is not likely to happen because of scarcity of the molecule even though an electron transfer to NBT is induced by the hole transfer to surplus MBA. 


\section{Does the Decarboxylation of MBA Occur Simultaneously with the Reduction of NBT in a Mixed SAM of MBA and NBT upon Plasmon Excitation?}

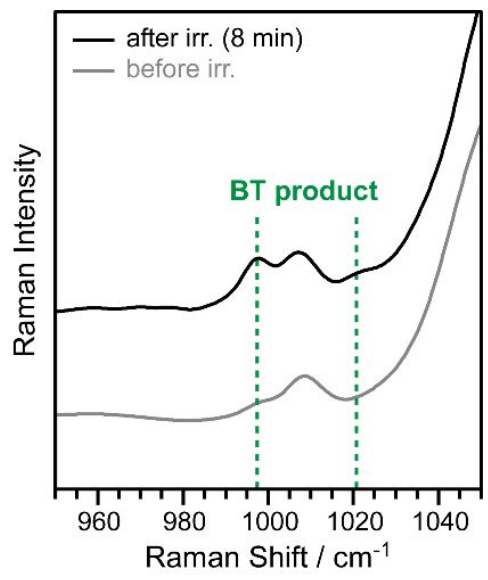

Figure S7. SERS spectra of mixed SAM of NBT and MBA in the nanogaps before and after irradiation (785 nm, $15 \mathrm{~mW}, 8 \mathrm{~min}$ ). The BT spectral region is enlarged to confirm that the decarboxylation of MBA, as well as DMAB formation from NBT, has occurred.

\section{Calculation of Product Yields}

(a) BT

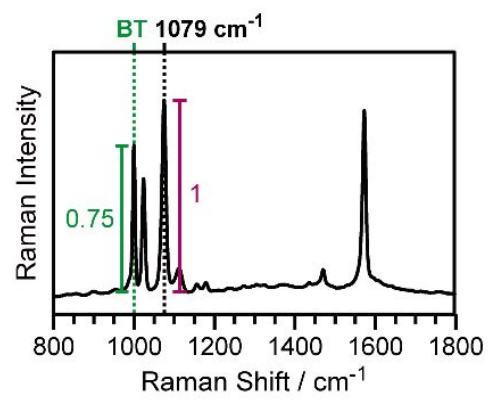

(b) $\mathrm{MBA} \rightarrow \mathrm{BT}$

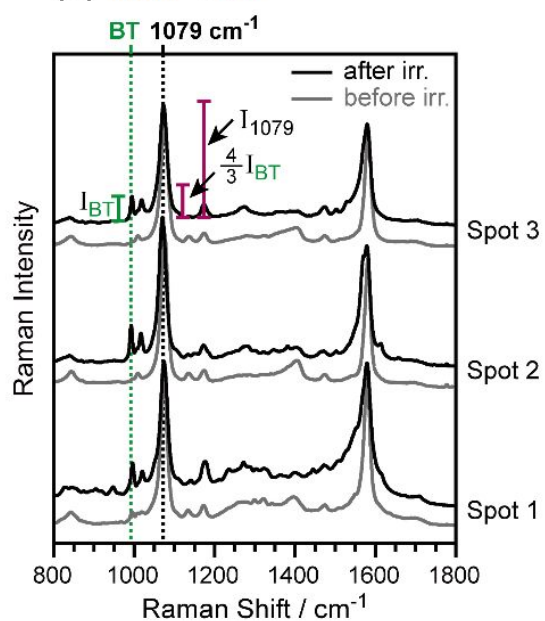

(c) NBT : MBA $=1: 9$

BT $1079 \mathrm{~cm}^{-1}$ NBT

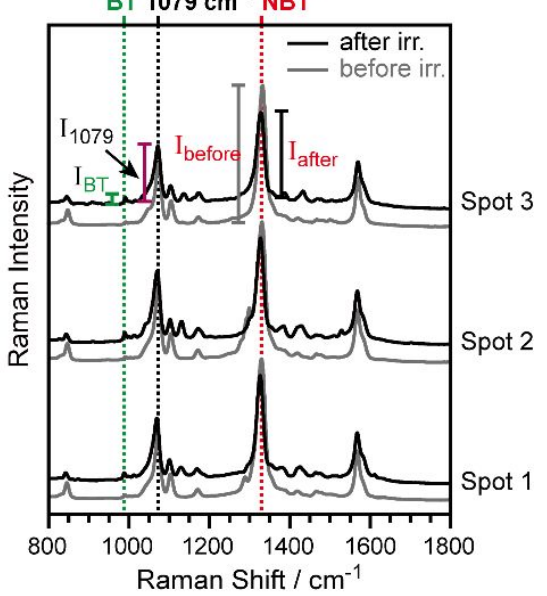

Figure S8. (a) SERS spectrum of BT in NPoM systems. "BT" marks the characteristic vibrational frequency of BT at $999 \mathrm{~cm}^{-1}$. "1079 $\mathrm{cm}^{-1}$ " indicates the position of the benzene ring in-plane bending mode $\left(\delta_{\mathrm{ccc}}\right)$, common to most aromatic compounds. (b) SERS spectra of MBA in NPoM before (gray lines) and after (black lines) irradiation at $785 \mathrm{~nm}$. (c) SERS spectra of NBT + MBA mixed SAMs in NPoM systems (mixing ratio, NBT:MBA = 1:9) before (gray lines) and after (black lines) irradiation. "NBT" denotes the characteristic vibrational frequency of NBT at $1340 \mathrm{~cm}^{-1}$. 
We used the high sensitivity and vibrational fingerprinting capability of SERS to measure the product yields from plasmon-driven reactions in NPoM. It is straightforward to calculate the yield for the formation of BT from MBA in NPoM. We first measured SERS spectra of BT in NPoM structures (Figure S8a). The characteristic vibrational frequency of BT appears at $999 \mathrm{~cm}^{-1}$, which we label as "BT". The common benzene ring in-plane bending mode $\left(\delta_{\mathrm{ccc}}\right)$ is observed at $1079 \mathrm{~cm}^{-1}$. The peak intensity ratio between the "BT" mode and $1079 \mathrm{~cm}^{-1}$ mode corresponds to 3:4.

To measure the BT product yield from the plasmon-driven decarboxylation reaction of MBA (MBA $\rightarrow$ BT), we obtained the SERS spectra of MBA in NPoM before and after irradiation at $785 \mathrm{~nm}$ (Figure S8b). BT forms from the reaction and small product peaks appear at $999 \mathrm{~cm}^{-1}$ with an intensity of $I_{\text {BT }}$. Unlike the $999 \mathrm{~cm}^{-1}$ peak, the $1079 \mathrm{~cm}^{-1}$ peak $\left(\mathrm{I}_{1079}\right)$ contains contributions from both unreacted remaining MBA and product BT. Because the Raman scattering cross-section of MBA and BT is almost identical (data not shown), ${ }_{3}^{4} \mathrm{I}_{\mathrm{BT}}$ constitutes the total peak intensity at $1079 \mathrm{~cm}^{-1}$ and the rest part of the intensity comes from unreacted MBA. Thus, the BT product yield corresponds to ${ }_{3}^{4} \mathrm{I}_{\mathrm{BT}} / \mathrm{I}_{1079}$. The calculations from several spectra acquired at different spots yield $32 \pm 2 \%$, which is consistent with our previous results (34 $\pm 2 \%){ }^{1}$

The reaction from the NBT + MBA mixed SAMs is a little more complicated (Figure S8c). For the yield for $\mathrm{BT}$, we first have to figure out how much contribution MBA gives to the $1079 \mathrm{~cm}^{-1}$ peak. Before the reaction, there are 9 times more MBA molecules than NBT molecules $\left(\mathrm{N}_{\mathrm{NBT}}: \mathrm{N}_{\mathrm{MBA}}=1: 9\right)$ in the probe spot. However, the Raman scattering cross-section of MBA is 8.8 times smaller than that of NBT $\left(\sigma_{\mathrm{NBT}}: \sigma_{\mathrm{MBA}}=\right.$ 8.8:1) (Figure S5). Because the Raman peak intensity is proportional to the number of molecules and the Raman scattering cross section $(I=N \cdot \sigma)$, NBT and MBA takes up 8.8/17.8 and $9 / 17.8$, respectively, of the total peak intensity $I_{1079}$. We find that the peak intensity at $1079 \mathrm{~cm}^{-1}$ remains largely unchanged after the reaction, suggesting that the Raman scattering cross-section of the product from NBT (i.e., DMAB) is about the same as that of NBT (we already know that the BT and MBA have the same cross-section). ${ }^{2}$ Thus, from

\footnotetext{
${ }^{1}$ Huh, H.; Trinh, D. H.; Lee, D.; Yoon, S. How Does a Plasmon-Induced Hot Charge Carrier Break a C-C Bond? ACS Appl. Mater. Interf. 2019, 11, 24715-24724.

2 One should note that two NBT molecules form one DMAB. Thus, the Raman scattering cross-section of DMAB should be compared with twice the Raman scattering cross-section of NBT.
} 
the SERS spectra after the reaction, $\frac{9}{17.8} \mathrm{I}_{1079}$ can be allotted to the contribution from MBA. Then, the BT product yield can be calculated by ${ }^{\frac{4}{3} \mathrm{I}_{\mathrm{BT}}} / \frac{9}{17.8} \mathrm{I}_{1079}$, yielding $23 \pm 5 \%$.

The DMAB yield can be estimated by measuring the decrease in the peak intensity of NBT. We measured the peak intensity at $1340 \mathrm{~cm}^{-1}\left(v_{\text {NOO }}\right.$ mode) before and after the irradiation. The ratio, $\frac{I_{\text {before }}-I_{\text {after }}}{I_{\text {before }}}$, provides the DMAB yield from NBT, corresponding to $29 \pm 5 \%$.

\section{Unreactive Molecules to Plasmon Excitation}

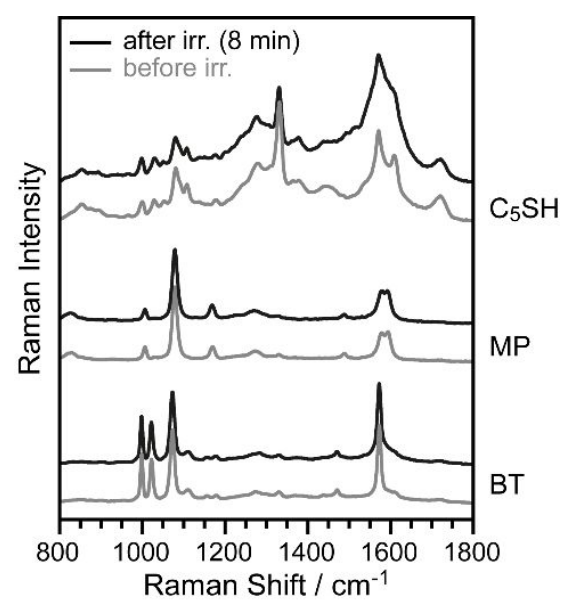

Figure S9. SERS spectra of pure BT, MP, and $\mathrm{C}_{5} \mathrm{SH}$ SAMs between AuNPs and Au films before and after irradiation $(785 \mathrm{~nm}, 15 \mathrm{~mW}, 8 \mathrm{~min})$. The SERS spectra remain unchanged despite irradiation, indicating that no plasmon-driven reaction occurs to these molecules. When these molecules are mixed with NBT, they do not switch on the NBT-to-DMAB reaction (Figure 6). 